\title{
Molecular beam study of dissociative sticking of methane on $\mathrm{Ni}(100)$
}

\author{
Holmblad, Peter Mikal; Wambach, Jørg; Chorkendorff, Ib
}

Published in:

Journal of Chemical Physics

Link to article, DOI:

$10.1063 / 1.468955$

Publication date:

1995

Document Version

Publisher's PDF, also known as Version of record

Link back to DTU Orbit

Citation (APA):

Holmblad, P. M., Wambach, J., \& Chorkendorff, I. (1995). Molecular beam study of dissociative sticking of methane on Ni(100). Journal of Chemical Physics, 102(20), 8255-8263. https://doi.org/10.1063/1.468955

\section{General rights}

Copyright and moral rights for the publications made accessible in the public portal are retained by the authors and/or other copyright owners and it is a condition of accessing publications that users recognise and abide by the legal requirements associated with these rights.

- Users may download and print one copy of any publication from the public portal for the purpose of private study or research.

- You may not further distribute the material or use it for any profit-making activity or commercial gain

- You may freely distribute the URL identifying the publication in the public portal

If you believe that this document breaches copyright please contact us providing details, and we will remove access to the work immediately and investigate your claim 


\title{
Molecular beam study of dissociative sticking of methane on $\mathrm{Ni}(100)$
}

\author{
P. M. Holmblad, J. Wambach, and I. Chorkendorff \\ Physics Department, Technical University of Denmark, DK-2800 Lyngby, Denmark
}

(Received 24 October 1994; accepted 16 February 1995)

\begin{abstract}
This paper reports a detailed molecular beam study of the dissociative sticking of methane incident on clean $\mathrm{Ni}(100)$. It is demonstrated that the sticking coefficient depends strongly on the translational energy of the molecule. It is also observed that an increase in the vibrational energy of the methane leads to a dramatic enhancement of the sticking, emphasizing that the initial vibrational state is of crucial importance. These results are consistent with a mechanism of direct activated dissociative chemisorption where the dynamics is dominated by a barrier in the potential energy surface mainly located in the vibrational coordinates. Normal-energy scaling is only approximately observed. The effect of surface temperature is also investigated but is found to be smaller than activation by translational or vibrational energy. A simplified analysis in terms of state resolved sticking curves, $S_{\nu}\left(E_{i}\right)$, considering only the vibrational C-H stretch modes of methane as relevant for the sticking, gives a good description of the data. These sticking curves enables a calculation of the thermal sticking coefficient which is found to be in excellent agreement with bulb experiments directly probing this. () 1995 American Institute of Physics.
\end{abstract}

\section{INTRODUCTION}

Methane dissociation is very important in the chemical industry. The steam-reforming process: $\mathrm{CH}_{4}+\mathrm{H}_{2} \mathrm{O} \rightarrow \mathrm{CO}$ $+3 \mathrm{H}_{2}$, where natural gas together with steam is transformed to synthesis gas (carbon monoxide and hydrogen) over supported nickel catalysts, is often the first step in a chain of heterogeneous catalytic processes since more complicated chemical products are then synthesized from $\mathrm{CO}$ and $\mathrm{H}_{2}{ }^{1}$ The rate limiting step in the steam reforming process is usually the dissociative chemisorption of methane on the nickel catalyst. Much effort has been put into the investigation of this fundamental sticking process which belongs to the class of activated dissociative chemisorption.

Methane chemisorption on metal surfaces has previously been studied with molecular beam methods on $\mathrm{Ni}(111)$, $\mathrm{Ni}(100), \operatorname{Pt}(111)$, and $\mathrm{W}(110){ }^{2-6}$ In these studies it was observed that the dissociation probability of the incident methane increased substantially with the initial translational energy associated with the velocity perpendicular to the surface. Also, it was found that vibrational excitation of the incident $\mathrm{CH}_{4}$ molecules plays some role in the process since the sticking probability was enhanced as the mean vibrational energy was increased. Finally a dependence on surface temperature has also been observed on the $\operatorname{Pt}(111)$ surface. ${ }^{4}$

Direct measurements of the thermal sticking coefficient have also been carried out for $\mathrm{Ni}(111), \mathrm{Ni}(110), \mathrm{Ni}(100),{ }^{7}$ and $\mathrm{Ni}(100) .{ }^{8}$ Nearly the same activation energy, $E_{a} \approx 50$ $\mathrm{kJ} / \mathrm{mol}$, has been observed for all three surfaces with $\mathrm{Ni}(110)$ and $\mathrm{Ni}(111)$ being the most and least reactive, respectively. However, some discrepancy exist for the $\mathrm{Ni}(100)$ surface

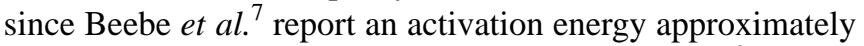
half the one later measured by Chorkendorff et al. ${ }^{8}$ Recent thermal activation experiments with $\mathrm{CH}_{4}$ on $\mathrm{Ni}(100)$ carried out in our laboratory are in agreement with the measurements of Chorkendorff et al. and will be reported separately. ${ }^{9}$

A subject of some debate is to what extent molecular beam experiments reveal the dynamics important at the con- ditions present in industrial catalytic processes. Is the sticking dominated by surface temperature dependent "precursor" dynamics or is it direct chemisorption depending principally on the gas temperature? For the $\mathrm{Ni}(111)$ surface reasonable agreement between thermal rates obtained from molecular beam results ${ }^{2}$ and bulb experiments ${ }^{7}$ suggest that this process is direct under thermal conditions. Also on the $\mathrm{Ni}(111)$ surface definite evidence for a direct process was found by Hanley et al. ${ }^{10}$ However, the previous molecular beam study on $\mathrm{Ni}(100)^{3}$ was not compatible with the thermal experiments $^{7,8}$ if the direct process in the beam experiment was assumed to also dominate in the thermal experiments. It has been suggested that the previous molecular beam study could be in error. ${ }^{7,11}$

In the present paper we report a new detailed molecular beam study of the dissociative sticking of $\mathrm{CH}_{4}$ incident on $\mathrm{Ni}(100)$. The importance of translational energy, angle of incidence, vibrational excitation, and surface temperature are investigated separately over a large range of these parameters. It is observed that the sticking probability depends strongly on the translational energy of the incident molecule giving a 2-3 orders of magnitude increase in the sticking coefficient as the translational energy is raised from 20 to $120 \mathrm{~kJ} / \mathrm{mol}$. Also it is observed that the vibrational temperature of the incident methane is of crucial importance since the sticking probability at a fixed translational energy of 40 $\mathrm{kJ} / \mathrm{mol}$ increases 2 orders of magnitude as the vibrational temperature is raised from 550 to $1050 \mathrm{~K}$. Normal-energy scaling is only approximately observed, the sticking probability at off-normal incidence being slightly lower than at normal incidence. The sticking probability is also increased as the surface temperature is raised, but this effect is found to be much weaker than the activation by translational and vibrational energy. The results clearly demonstrate that in molecular beam experiments on activated dissociative chemisorption, it is of vital importance to investigate the role of translational energy, vibrational temperature, and surface temperature separately. 


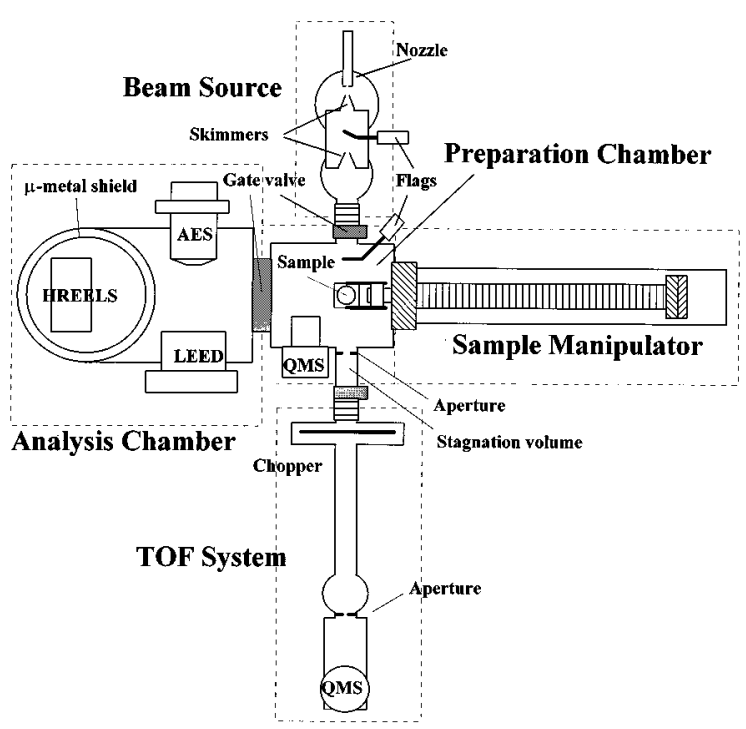

FIG. 1. Schematic of the UHV apparatus and molecular beam line used in this work to measure sticking coefficients.

This detailed molecular beam study provides strong evidence for a mechanism of activated direct chemisorption, where the barrier to dissociation in the potential energy surface (PES) governing the dynamics is mainly located in the vibrational coordinates. Also this study is in excellent agreement with the dynamical model of direct chemisorption proposed by Luntz et al., ${ }^{12,13}$ where methane is treated as a quasidiatomic molecule and the surface temperature dependence is a consequence of thermally assisted tunneling. This comparison is not a proof of that particular model, but the model allows for a plausible and transparent interpretation of the obtained results of the present paper.

A parametrization of the data in terms of vibrational state resolved sticking curves provides a good description of the data. The extrapolation of these sticking curves to low energies enables a calculation of the thermal sticking coefficient which is in very good agreement with the direct measurements of Chorkendorff et al. ${ }^{8}$

\section{APPARATUS}

The apparatus used for these studies consists of a stainless steel ultra high vacuum (UHV) chamber combined with a supersonic molecular beam line with time of flight (TOF) analysis. The system is schematically shown in Fig. 1.

A large gate valve divides the main chamber in an analysis section and a preparation section. The analysis section is equipped with Auger electron spectroscopy (AES), low energy electron diffraction (LEED), and electron energy loss spectroscopy (EELS). This section is pumped by a $500 \ell / \mathrm{s}$ ion pump.

On the preparation section there is a sputter gun and a quadrupole mass spectrometer (QMS) for residual gas analysis and thermal desorption spectroscopy. A number of leak valves connected to a gas handling system allow the sample to be exposed to various gases at moderate pressures $(p<10$ mbar). The preparation section is pumped with a $450 \ell / \mathrm{s}$ turbo molecular pump (TMP) which can be isolated from the chamber with a pneumatic gate valve. The main chamber base pressure is $p_{\text {base }} \sim 7-8 \times 10^{-11}$ mbar.

A horizontal manipulator translates the sample along the chamber axis and allows also for perpendicular motions, rotation, and tilting. The sample is fixed on two tungsten wires and can be resistively heated using a low voltage power supply. The sample temperature is measured with a cromelalumel thermocouple positioned in a spark cut hole in the sample.

The molecular beam line goes through the preparation section perpendicular to the chamber axis. The molecular beam source consists of a molybdenum nozzle with an aperture of $40 \mu \mathrm{m}$. It is water cooled and can be resistively heated from 290 to $1100 \mathrm{~K}$. The temperature is measured with a cromel-alumel thermocouple spot welded to the nozzle. Gas is supplied to the nozzle from a gas handling system where gas mixtures can be prepared and cleaned by catalysts prior to the expansion. Stagnation pressures behind the nozzle can be controlled from 0.1 to 7.0 bar. The gas is expanded through the nozzle aperture into an exhaust chamber pumped with a $450 \ell / \mathrm{s}$ TMP. The beam is extracted with a skimmer $(D=0.2 \mathrm{~mm})$ on the center line of the expansion approximately $2.5 \mathrm{~mm}$ from the nozzle aperture. It then enters a second pumping stage $(180 / / \mathrm{s}$ TMP $)$ and a second collimating skimmer $(D=0.5 \mathrm{~mm}) 115 \mathrm{~mm}$ from the nozzle defines the solid angle of the beam. The molecular beam then passes a third pumping stage (180 $\ell / \mathrm{s}$ TMP) before finally entering the preparation chamber.

On the opposite side of the preparation section, the supersonic molecular beams are characterized with a TOF system. This consists of a single slit $(1.4 \mathrm{~mm})$ chopper disk $(D=22 \mathrm{~cm})$, a QMS with cross beam ionization, and a PC with a fast multichannel analyzer (MCA). The MCA is triggered by a fast diode/phototransistor system detecting the rotation of the chopper disk. The chopper can be operated in the range $30-300 \mathrm{~Hz}$ and the flight path from chopper to ionization is $110 \mathrm{~cm}$. The TOF system is pumped with two $120 \ell / \mathrm{s}$ ion pumps and a $60 \ell / \mathrm{s}$ TMP.

On the beam line just before the TOF system, the beam can be blocked with a gate valve to establish a $0.3 \ell$ stagnation volume where pressure can be measured with an ion gauge. The circular entrance aperture to the stagnation volume has a diameter of $4.5 \mathrm{~mm}$.

\section{EXPERIMENT}

The circular Ni(100) sample used in this study has a thickness of $2.5 \mathrm{~mm}$. The crystal was spark cut from a single crystal nickel rod aligned by Lane backreflection x-ray diffraction and diamond polished. It was oriented to within $0.5^{\circ}$ of the (100) face. In the UHV chamber the sample was initially cleaned by several cycles of sputtering $\left(2 \mathrm{kV} \mathrm{Ar}^{+}\right)$and oxidation at $1000{ }^{\circ} \mathrm{C}\left[10\right.$ times $5 \mathrm{~min}$ at $p\left(\mathrm{O}_{2}\right)=5 \times 10^{-7}$ mbar]. After this procedure all impurities were below the detection limit of AES and the surface showed a sharp $p(1 \times 1)$ LEED pattern. Surface carbon resulting from dissociation of methane could then routinely be removed by 


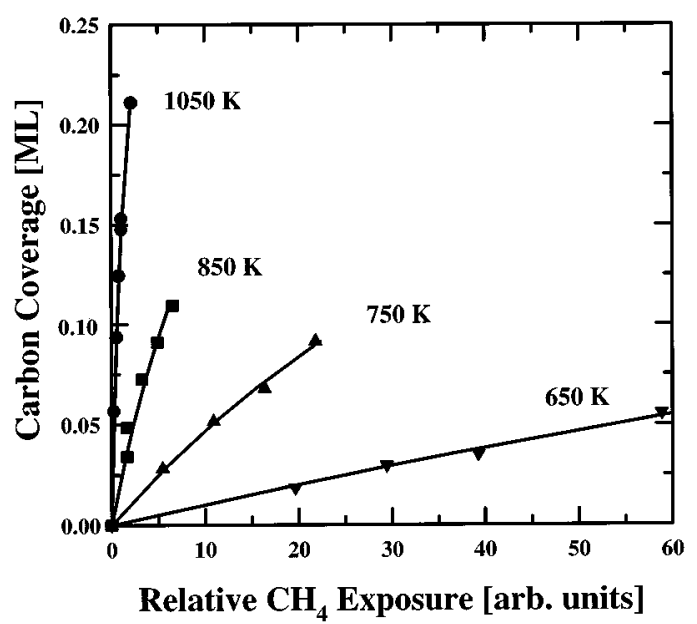

FIG. 2. Examples of carbon uptake obtained with a beam of fixed gas composition $\left(10 \% \mathrm{CH}_{4}\right.$ in $\left.\mathrm{He}\right)$ at various nozzle temperatures as indicated.

gentle oxidation $\left[p\left(\mathrm{O}_{2}\right)=1 \times 10^{-7} \mathrm{mbar}\right.$ at $800{ }^{\circ} \mathrm{C}$ for $\left.30 \mathrm{~s}\right]$ and subsequent reduction of excess oxygen with hydrogen at $700{ }^{\circ} \mathrm{C}\left[p\left(\mathrm{H}_{2}\right)=1 \times 10^{-6} \mathrm{mbar}\right.$ for $\left.8 \mathrm{~min}\right]$.

Measurements of the sticking probability versus normal energy were carried out with beams of several initial compositions $\left(3 \%, 10 \%, 25 \%\right.$, and $50 \% \mathrm{CH}_{4}$ in $\mathrm{He}, 3 \% \mathrm{CH}_{4}$ in $\mathrm{H}_{2}$ and pure $\mathrm{CH}_{4}$ ). Also $3 \% \mathrm{CD}_{4}$ in $\mathrm{He}$ was used to investigate the isotope effect. The $3 \% \mathrm{CH}_{4}$ and $\mathrm{CD}_{4}$ in He were supplied with purity N55. Other gas compositions were mixed on the molecular beam gas handling system from pure $\mathrm{CH}_{4}(\mathrm{~N} 55)$, $\mathrm{He}(\mathrm{N} 60)$, and $\mathrm{H}_{2}(\mathrm{~N} 60)$ and stored in a stainless steel vessel. $\mathrm{CH}_{4} / \mathrm{He}$ mixtures were further purified prior to expansion by passing the gas through a freshly reduced nickel catalyst at room temperature. For the $\mathrm{CH}_{4} / \mathrm{H}_{2}$ mixture a copper catalyst was used to purify the mixture.

Sticking probabilities were obtained from the carbon uptake on the surface versus molecular beam exposure of methane. During an exposure, all hot filaments in the preparation chamber were turned off. The determination of the initial sticking coefficient involved typically 3-5 measurements at various coverages. Some examples are shown in Fig. 2 where a beam of $10 \% \mathrm{CH}_{4}$ in $\mathrm{He}$ was used. With the sticking coefficient as the only free parameter, the model uptake curve used in Ref. 8 was fitted to the data to account for the coverage dependence of the sticking coefficient. This uptake expression is derived from a model of random chemisorption on sites with free neighbor sites ${ }^{14}$ and provided an accurate description of the carbon uptake at coverages lower than 0.3 ML $\left(1 \mathrm{ML}=1.6 \times 10^{15} \mathrm{~cm}^{-2}\right)$.

Above a coverage of $0.30-0.35 \mathrm{ML}$ the surface saturates with carbon faster than the predictions of this random chemisorption model. This is in agreement with a recent STM study of ethylene decomposition on $\mathrm{Ni}(100),{ }^{15}$ where it was observed that surface carbon induced reconstructed $(2 \times 2)$ patches with the $p 4 g$ symmetry at a local coverages above $0.2 \mathrm{ML}$. The consequence of this reconstruction is more free nickel surface area available for sticking compared to the site blocking following random chemisorption. The local reconstruction is observed in the present study to significantly influence the model at coverages higher than $\sim 0.3 \mathrm{ML}$.
In this molecular beam study, the crystal was kept at $T_{S}=475 \mathrm{~K}$ so that after chemisorption methane is decomposed completely to surface carbon followed by recombinative desorption of hydrogen.

The carbon coverages were determined with AES using an electron beam current of $1.5 \mu \mathrm{A}$ at $3 \mathrm{keV}$. The carbon coverage was assumed proportional to the differentiated Auger $\mathrm{C}(270 \mathrm{eV})$ and $\mathrm{Ni}(848 \mathrm{eV})$ peak ratio. Calibration of this ratio was performed by exposing the surface to ethylene at $475 \mathrm{~K}$ until saturation. The ratio $[\mathrm{C}(270 \mathrm{eV}) / \mathrm{Ni}(848 \mathrm{eV})]_{\text {sat }}$ was found to 0.26 . At this coverage the characteristic LEED pattern corresponding to the $p 4 g$ symmetry was observed. The same ratio and LEED pattern was found when the sample was exposed to a high energy molecular beam of methane until saturation. This ratio corresponds to the saturation concentration of surface carbon known to be $\theta=0.5$ ML. ${ }^{16}$

Using AES, the diameter of the carbon spot on the $\mathrm{Ni}(100)$ surface after a beam exposure was found to be approximately $3 \mathrm{~mm}$, consistent with the solid angle determined by the limiting second skimmer of the beam source.

Methane fluxes were determined by a combined use of the QMS signal of $m / e=16, I_{\mathrm{QMS}}$, in the preparation chamber, and the equilibrium pressures, $p_{S}$, obtained in the stagnation volume. The $I_{\mathrm{QMS}}$ signal provides a relative measurement of the flux of methane in the beam. However, for experimental convenience $p_{S}$ was used instead and for each gas mixture a linear relation between $p_{S}$ and $I_{\mathrm{QMS}}$ was determined experimentally. Using $I_{\mathrm{QMS}}$ as a measure of the methane flux in the beam, relative sticking probabilities were obtained with the Auger method.

The absolute magnitude of the sticking coefficients was calibrated using measurements based on the method of King and Wells. ${ }^{17}$ This method provides an absolute measurement of the sticking coefficient and could be applied at sticking coefficients higher than $\sim 0.03$. A calibration factor relating the absolute flux of methane in the beam to $I_{\mathrm{QMS}}$ could then be found by comparing the absolute sticking coefficient obtained using the method of King and Wells to the relative sticking coefficient simultaneously obtained from the carbon uptake using $I_{\mathrm{QMS}}$ as a measure of the flux.

All beams were characterized using TOF analysis. The beam velocities were modeled by a shifted Maxwellian distribution. Using nonlinear curve fitting the TOF spectra obtained with a density sensitive detector were therefore analyzed according to

$$
g(t) d t \propto v^{4} \exp \left[-\frac{(1 / 2) W_{i}\left(v-v_{0}\right)^{2}}{R T_{i}}\right] d v, \quad v=\frac{L}{t},
$$

where $W_{i}$ is molar weight, $R$ is the gas constant and, $v_{0}$ and $T_{i}$ are parameters to be determined. The true flight time over the distance, $L$, from the chopper to the ionization is $t=t_{\mathrm{TOF}}-\delta t_{\text {trig }}-\delta t_{\mathrm{QMS}}$. Here, $t_{\mathrm{TOF}}$ is the apparent flight time from the TOF spectrum and $\delta t_{\mathrm{QMS}}$ and $\delta t_{\text {trig }}$ are delays due to triggering and detection, respectively.

Delays due to time spent in the QMS detection depends on the mass of the species. This was modeled according to $\delta t_{\mathrm{QMS}}=K \sqrt{m / e}$, where the constant $K$ involves parameters 


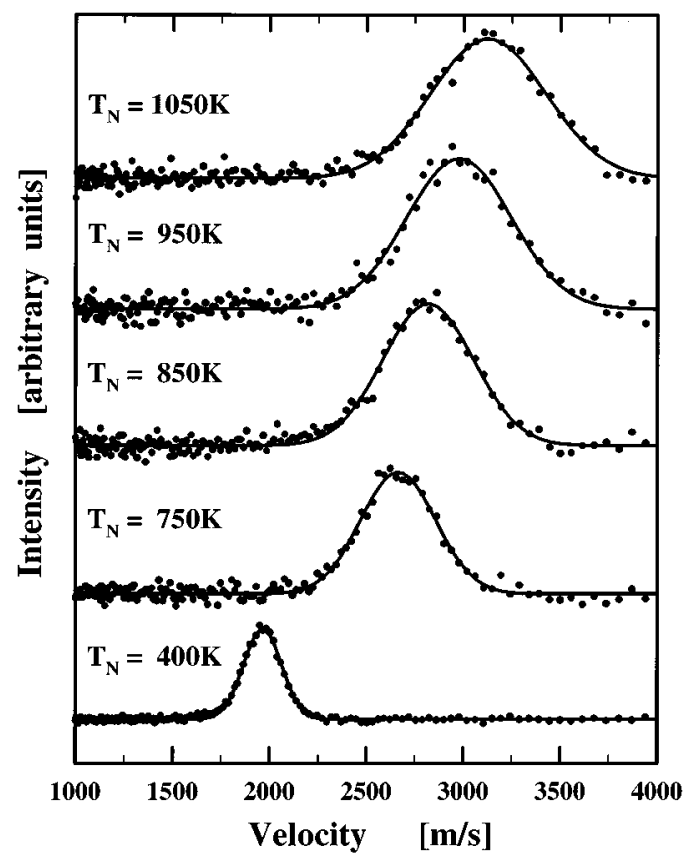

FIG. 3. Examples of velocity distribution measured by TOF for a gas mixture of $3 \% \mathrm{CH}_{4}$ in $\mathrm{He}$ at various nozzle temperatures.

of the detection such as the flight path and potentials in the QMS. This constant was determined from the cracking pattern of methane. The trigger offset, $\delta t_{\text {trig }}$, was determined by adjusting flight times of a pure helium beam at various temperatures to fit those theoretically expected for this monoatomic noble gas. In this way the beam of pure helium is used as a reference beam. The beams were characterized using a $5 \mu \mathrm{s}$ resolution for the MCA and a chopper frequency of $100 \mathrm{~Hz}$.

In all the experiments, the expansion was controlled so that the pressure in the exhaust chamber was kept constant at $4 \times 10^{-3}$ mbar. Depending on gas composition and nozzle temperature, this required the stagnation pressures to be adjusted in the range $0.5-7$ bar.

All beams could be accurately described by expression (1). The typical broadening in the TOF expressed as full width at half-maximum (FWHM) was $\delta E / E=0.35-0.40$, although the broadening was somewhat larger at the highest nozzle temperatures. Figure 3 shows an example of the measured velocity distributions as the nozzle temperature is increased for a beam with initial gas composition of $3 \% \mathrm{CH}_{4}$ in He.

The supersonic molecular beam source produces a beam of methane molecules with a fairly narrow translational kinetic energy distribution. The mean energy is approximately given by

$$
E_{\mathrm{CH}_{4}}=W_{\mathrm{CH}_{4}} \frac{\left\langle C_{p}\right\rangle}{\langle W\rangle} T_{N},
$$

where $\left\langle C_{p}\right\rangle=R \Sigma_{i} X_{i} \gamma_{i} /\left(\gamma_{i}-1\right)$ is the average molar heat capacity, $\langle W\rangle=\Sigma_{i} X_{i} W_{i}$ is the average molar weight of the gas, and $T_{N}$ is the temperature of the nozzle. $X_{i}$ and $\gamma_{i}$ designate mole fraction and heat capacity ratio, respectively. The trans- lational energy of the methane can therefore be controlled either by varying the gas composition and/or the nozzle temperature.

The population of the internal degrees of freedom depends on the nozzle temperature. These levels are just prior to the expansion populated according to a Boltzmann equilibrium distribution at the nozzle temperature. Energy relaxation of the vibrational levels in methane is very ineffective compared to relaxation of energy in the translational degrees of freedom. Since the density in the adiabatic expansion is decreasing very fast there will not be enough inelastic collisions for the methane to significantly cool vibrationally. To a good approximation it can be assumed that no relaxation occurs at all. Therefore, the vibrational levels of methane molecules approaching the $\mathrm{Ni}(100)$ surface are assumed populated according to a Boltzmann distribution at the nozzle temperature used.

Energy relaxation in the rotational states of methane is indirectly observed to be complete. When the final translational and rotational temperature, $T_{i}$, of the gas component $i$ are taken into account Eq. (2) modifies to ${ }^{18}$

$$
\sum_{i} X_{i} \frac{1}{2} W_{i} v_{i}^{2}=\sum_{i} X_{i} \frac{\gamma_{i}}{\gamma_{i}-1} R\left(T_{N}-T_{i}\right) .
$$

The final temperature is found from the width of the velocity distribution as measured by TOF. In Eq. (3) it is assumed that for each gas component the temperature parallel and perpendicular to the beam direction are the same. When expression (3) is fitted to the measured energies and final temperatures obtained with beams of pure methane, the value of $\gamma_{\mathrm{CH}_{4}}$ is found to 1.34. This is the value of $\gamma$ expected if three translational and three rotational degrees of freedom are contributing to the heat capacity. Also, Eq. (3) accounts very well for all the measured beam energies obtained with different initial $\mathrm{CH}_{4} / \mathrm{He}$ gas compositions and nozzle temperatures, when $\gamma_{\mathrm{CH}_{4}}=1.34$, and $\gamma_{\mathrm{He}}=1.67$ are used in the calculation of theoretical beam energies. For hydrogen $\gamma_{\mathrm{H}_{2}}$ is found to $\sim 1.6$.

In the experiments reported here the translational and vibrational energy of the beam was controlled by separately varying initial gas composition and nozzle temperature.

Considerable care was taken to ensure the absence of artifacts in the experiments. Control experiments were carried out in order to examine the quality of the UHV, the purity of the gasses and chemistry going on in the nozzle at the rather elevated temperatures used in the experiments.

(1) The crystal was kept at exposure conditions without having the beam on for periods much longer than the exposure times used for measurements. No carbon build up could be detected on the crystal surface.

(2) Same as (1) with the beam directed into the preparation chamber but not incident on the crystal surface. Here, a very small background influence was observed corresponding to $\approx 0.001 \mathrm{ML}$ per $\mathrm{h}$. Carbon coverages obtained after very long exposures are corrected for this small background adsorption.

(3) The crystal was exposed to a $100 \%$ methane beam at $1050 \mathrm{~K}$ nozzle temperature. This low energy beam $\left(E_{i} \approx 25\right.$ 


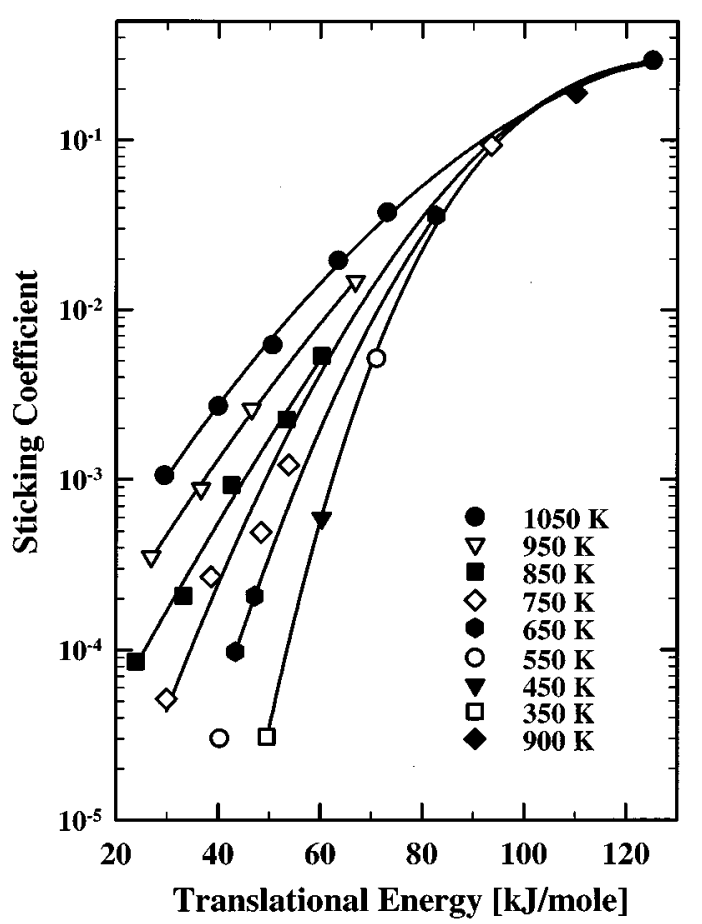

FIG. 4. Initial sticking coefficient at normal incidence as a function of translational energy at various vibrational temperatures as indicated by the symbol legend. The surface temperature is $475 \mathrm{~K}$. The lines through the points have no theoretical significance.

$\mathrm{kJ} / \mathrm{mol}$ ) was directed on to the crystal at $60 \mathrm{deg}$ off normal incidence for $2 \mathrm{~h}$. A very small sticking probability for methane is expected at this exposure condition since the normal energy of the incident methane is very low. However, the sticking of eventual impurities and highly reactive radicals formed at the high nozzle temperature will not be reduced. Only minor amounts of carbon $(\sim 0.015 \mathrm{ML})$ could be detected after this treatment. This corresponds to a sticking probability of less than $5 \times 10^{-5}$. Therefore, it can be concluded that only very few highly reactive radicals are formed at the high nozzle temperatures and that the gas contains only few contaminations which would have an adverse influence on the measured sticking coefficients.

(4) The crystal was exposed to a beam of pure helium expanded at $1050 \mathrm{~K}$ nozzle temperature for periods much longer than the exposure times used in the experiments. No carbon could be detected on the $\mathrm{Ni}(100)$ surface after this and any contaminations originating from the heated nozzle could be ruled out.

\section{RESULTS}

Figure 4 summarizes the measured sticking coefficients obtained using different initial gas compositions with the incident beam perpendicular to the $\mathrm{Ni}(100)$ surface. The figure shows how the sticking coefficient depends on the translational energy and the vibrational temperature of the methane at a constant surface temperature of $475 \mathrm{~K}$.

As has been observed earlier for $\mathrm{CH}_{4}$ incident on other metal surfaces, the sticking coefficient shows the characteristics of activated direct chemisorption, i.e., a strong depen-

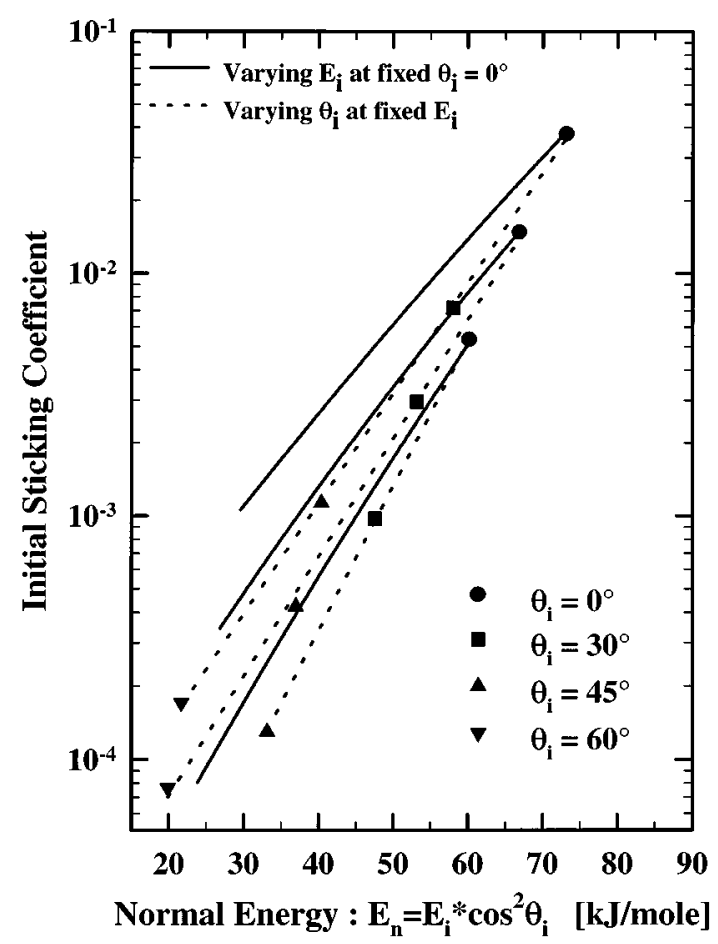

FIG. 5. Initial sticking coefficients as a function of normal energy measured at various angles of incidence as indicated by the symbol legend. The indicated temperatures are the vibrational temperatures. Solid lines are smooth curves through the sticking coefficients measured at normal incidence at the same vibrational temperatures.

dence on the translational energy. The sticking probability is increased between 2 and 3 orders of magnitude as the translational energy for fixed vibrational temperature is raised from 20 to $120 \mathrm{~kJ} / \mathrm{mol}$. When the nozzle temperature for a fixed gas composition is raised to increase both the translational as well as vibrational energy the increase is even more dramatic. The most novel feature of the $\mathrm{CH}_{4} / \mathrm{Ni}(100)$ system is the dramatic effect of vibrational excitation. For example, at a fixed translational energy of $40 \mathrm{~kJ} / \mathrm{mol}$ the sticking coefficient increases 2 orders of magnitude as the vibrational temperature is raised from 550 to $1050 \mathrm{~K}$. This vibrational enhancement of the sticking is substantially more pronounced than was observed on $\mathrm{W}(110)$ and $\mathrm{Pt}(111)$. The translational activation is of the same magnitude, but the comparison is somewhat complicated by the role of vibrational excitation.

Figure 5 shows how the sticking coefficient is influenced by angle of incidence $\theta$ from the surface normal. The energy scale $E_{n}=E_{i} \cos ^{2} \theta$ is the translational energy associated with the velocity normal to the surface. The solid lines are smooth curves through sticking coefficients from Fig. 4 obtained at normal incidence, where $E_{i}$ is varied for fixed $\theta=0^{\circ}$ at constant vibrational temperatures of 1050,950 , and $850 \mathrm{~K}$. Superimposed on these are sticking coefficients obtained at off-normal incidence using a beam of fixed gas composition ( $3 \% \mathrm{CH}_{4}$ in $\mathrm{He}$ ) where $E_{i}$ is fixed as $\theta$ is varied from $0^{\circ}$ to $60^{\circ}$ at the same vibrational temperatures. As can be seen simple normal-energy scaling is only approximately observed, the off-normal sticking probability systematically be- 


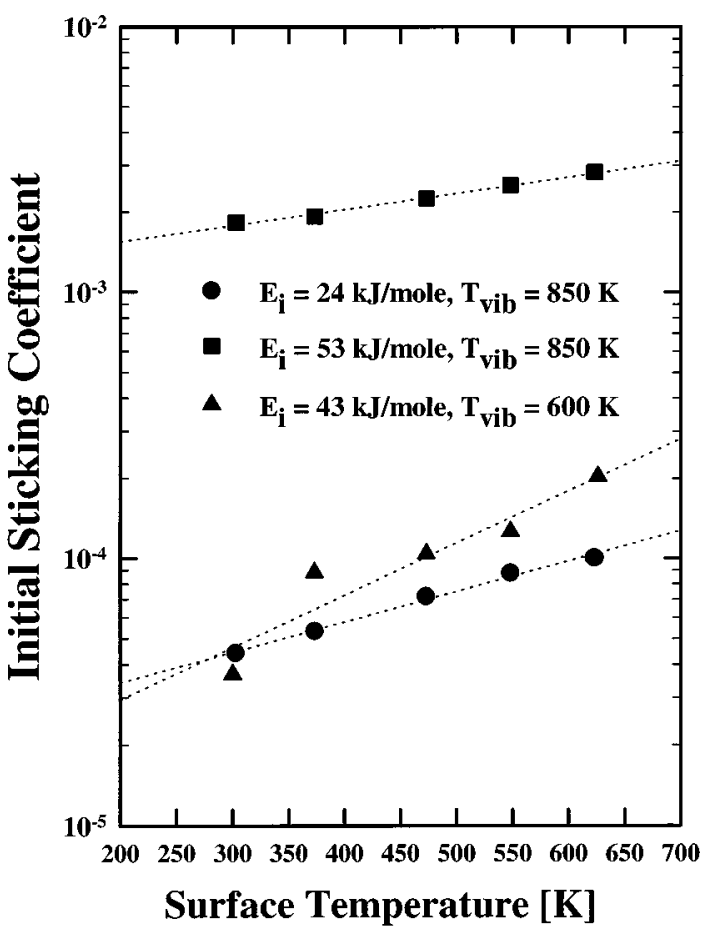

FIG. 6. Initial sticking coefficient as function of surface temperature at three different conditions of translational and vibrational energy.

ing slightly lower than expected for normal-energy scaling.

The effect of surface temperature was studied in the range 300 to $625 \mathrm{~K}$ at three different conditions of fixed translational energy and vibrational excitation. These results are shown in Fig. 6. The sticking coefficient depends only weakly on surface temperature at the experimental conditions. The effect is strongest at low translational energy and/or low vibrational temperature. The strongest effect observed with the $\mathrm{CH}_{4}$ beam having a translational energy of $43 \mathrm{~kJ} / \mathrm{mol}$ and a vibrational temperature of $600 \mathrm{~K}$. At these conditions the sticking coefficient increases by a factor of approximately 6 as the surface temperature is increased from 300 to $625 \mathrm{~K}$. Surface temperature effects are small compared to those of translational energy and vibrational excitation.

Figure 7 shows the sticking coefficient measured for $\mathrm{CH}_{4}$ and $\mathrm{CD}_{4}$ both expanded as 3\% methane in He. An isotope effect of approximately a factor of 5-10 is observed, $\mathrm{CH}_{4}$ being most reactive for the same translational energy.

\section{DISCUSSION}

The dissociation dynamics observed in this study qualitatively exhibits the features of the model proposed by Luntz et al., ${ }^{12}$ i.e., the process is direct dissociative chemisorption where a barrier in the PES separates the intact $\mathrm{CH}_{4}$ molecule from its chemisorbed fragments $\mathrm{CH}_{3}-*$ and $\mathrm{H}-*$. In this context the strong dependency on vibrational excitation of the incident molecule is the consequence of an "exit channel" barrier, that is a barrier mainly located in the vibrational coordinate. The activation by surface temperature is also in qualitative accordance with the mechanism of thermally as-

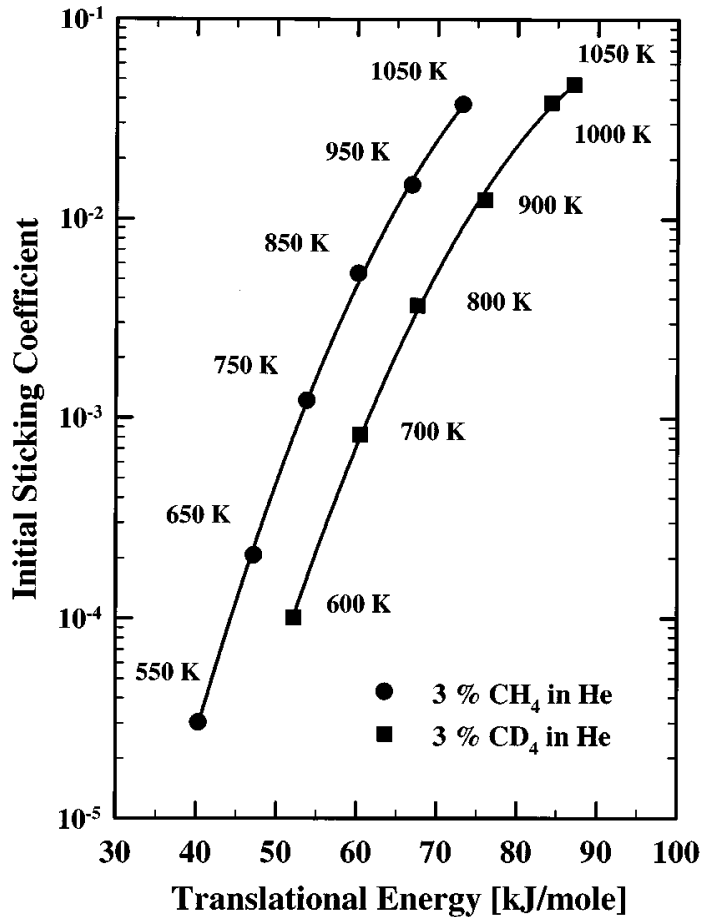

FIG. 7. Initial sticking coefficient at normal incidence of $\mathrm{CH}_{4}$ (solid circles) and $\mathrm{CD}_{4}$ (solid squares) as function of translational energy and vibrational temperature as indicated at the points.

sisted tunnelling proposed by Luntz et al. where the motion of the surface atoms is included in a simple "spring and ball" model and the sticking probability depends on the relative motion between the incident methane molecule and the surface atoms. Therefore, the surface temperature effect is most important at low translational energy and ceases at higher translational energies where the speed of the surface atoms becomes insignificant. Also the observed isotope effect is consistent with this model. A more detailed comparison will be given in the following paper. ${ }^{13}$

In Fig. 8 the sticking coefficient for constant translational energy is plotted versus inverse vibrational temperature. The translational energies are shown to the right of each curve. This Arrhenius plot corresponds to vertical cuts in the data shown in Fig. 4. The plot was constructed by fitting a smooth curve through measured sticking coefficients of constant vibrational temperature thereby eliminating the effect of energy distributions in the beams and enabling interpolation between the points. It is clearly seen that at low translational energies there is a strong activation by vibrational temperature, whereas this effect decreases as the translational energy increases. Although the plot does show curvature, which they should since more than one vibrational excitation are involved, an average activation energy can be determined by fitting straight lines. These activation energies are indicated in parentheses in Fig. 8 expressed in units of the vibrational quantum of the stretch modes in methane $\left(\hbar \omega_{0}=35\right.$ $\mathrm{kJ} / \mathrm{mol}$ ). In a model, where only $\mathrm{C}-\mathrm{H}$ stretch vibrations are considered important, this means that at low translational energies the sticking is dominated by methane molecules having two vibrational quanta in the $\mathrm{C}-\mathrm{H}$ stretch mode. As 


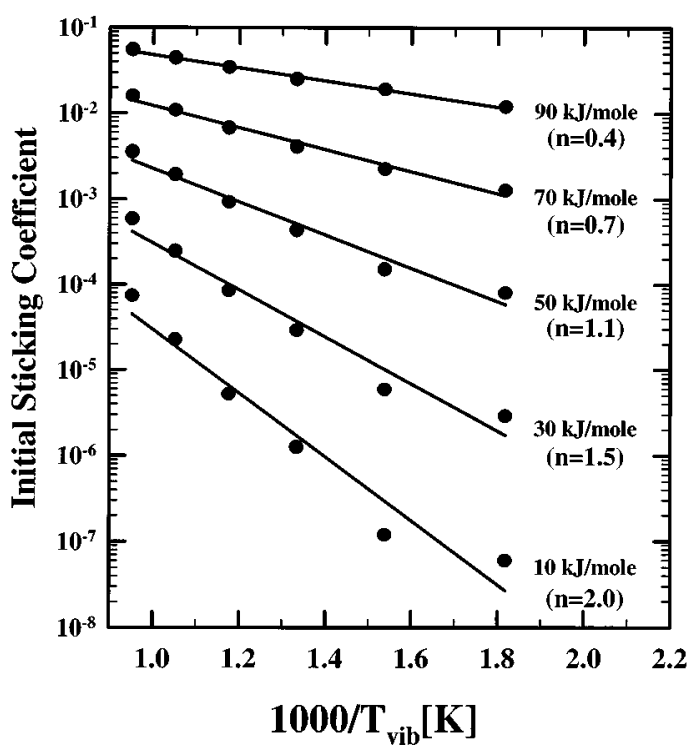

FIG. 8. Initial sticking coefficient as function of inverse vibrational temperature at various constant translational energies as indicated. The plot corresponds to vertical cuts in the data shown in Fig. 4. An average activation energy is shown in parenthesis in units of the energy quantum of the $\mathrm{C}-\mathrm{H}$ stretch mode of methane $(35 \mathrm{~kJ} / \mathrm{mol})$.

the translational energy is increased the sticking gradually becomes dominated by molecules with one vibrational quantum and at high translational energy the sticking is dominated by molecules in the vibrational ground state.

More detailed information about the PES governing the dynamics of the dissociative chemisorption can be extracted from the molecular beam data when these are interpreted in terms of state resolved sticking curves, that is smooth curves describing the dependency on translational energy of molecules in a particular vibrational state. These curves defines a threshold for the sticking, a broadening around this threshold and a saturation level. In principle, a sticking curve should be assigned to each distinguishable vibrational state. However, including all nine vibrational degrees of freedom in methane would result in a too complex model having too many free parameters. Following the ideas of Luntz et al., ${ }^{12}$ we believe that the only important vibrational excitation is that of $\mathrm{C}-\mathrm{H}$ stretches. Therefore, methane is approximated as a quasidiatomic molecule $\mathrm{H}-\mathrm{R}$ having a four times degenerate stretch vibrational mode to account for the fact that this quasidiatomic model represents $\mathrm{H}-\mathrm{CH}_{3}$. The population of vibrational state $\nu$ is

$$
f_{\nu}\left(T_{\mathrm{vib}}\right)=g_{\nu} \exp \left(-\frac{\nu \hbar \omega_{0}}{R T_{\mathrm{vib}}}\right)\left[1-\exp \left(-\frac{\hbar \omega_{0}}{R T_{\text {vib }}}\right)\right]^{4}
$$

where $g_{\nu}$ is the degeneracy $\left(g_{i}=1\right.$ for $\nu=0$ and $g_{i}=4$ for $\nu=1,2)$. At the nozzle temperatures used in these experiments only energy levels $\nu \cdot \hbar \omega_{0}$ with $\nu=0,1$, and 2 will be populated with sufficient weight to contribute to the magnitude of the sticking coefficients.

In the present work, the ansatz for the smooth " $\mathrm{S}$ "shaped sticking curve suggested by Michelsen et al. ${ }^{19}$ is used

$$
S_{\nu}(E)=A_{\nu}\left[1+\operatorname{erf}\left(\frac{E-V_{\nu}}{W_{\nu}}\right)\right]
$$

where erf is the error function. This is not a unique choice but allows for a plausible threshhold separation of the sticking curves as a function of vibrational excitation.

The normalized flux weighted translational energy distribution of the molecular beam is

$$
f\left(E_{i}, T_{N}\right)=E_{i} \exp \left[-\frac{\left(\sqrt{E_{i}}-\sqrt{E_{0}}\right)^{2}}{R T}\right],
$$

where the parameters $E_{0}$ and $R T$ depends on the nozzle temperature.

The sticking coefficient measured in the experiments is then given by the population weighted sum over relevant $S_{\nu}(E)$ convoluted with the translational energy distribution in the beam

$$
S_{\exp }\left(T_{N}\right)=\sum_{\nu=0}^{2} f_{\nu}\left(T_{N}\right) \int_{0}^{\infty} S_{\nu}(E) f\left(E, T_{N}\right) d E .
$$

Applying nonlinear curve fitting with numerical evaluation of the integrals, this parametrization with a maximum of nine parameters combined with various constraints relating these, is fitted to the measured sticking coefficients shown in Fig. 4. Nine adjustable parameters leaves some freedom in the fitting and careful attention was paid to the uniqueness of the fits. The parameter $W_{2}$ was not sufficiently well determined by the data and was therefore fixed during a fitting routine and successively adjusted to reasonable values when compared to values obtained for $W_{1}$ and $W_{0}$. The best physical reasonable parametrization with the chosen ansatz is obtained fixing all $A_{\nu}$ 's to one and fitting the remaining five parameters without constraints and adjusting $W_{2}$ as described. The alternating solid and dashed lines in Fig. 9 shows the result of this fit together with the measured sticking coefficients (symbols) corrected for the velocity distributions in the beams. The $S_{\nu}(E)$ curves obtained in this way are shown in Fig. 10. The parameters obtained are in $\mathrm{kJ} / \mathrm{mol}$ : $V_{0}=136.7, W_{0}=29.6, V_{1}=78.7, W_{1}=23.5, V_{2}=32.0$, $W_{2}=15.0$, and $A_{\nu}=1.0$ for all $\nu$. The uncertainties in these parameters are estimated to $\Delta V_{\nu} \approx 5 \mathrm{~kJ} / \mathrm{mol}, \Delta W_{\nu} \approx 2 \mathrm{~kJ} / \mathrm{mol}$ for $\nu=0,1$ and $\Delta W_{2} \approx 5 \mathrm{~kJ} / \mathrm{mol}$. It is seen that the threshold for dissociation is shifted down in translational energy and that the broadening around the thresholds is decreased as the molecule becomes vibrationally excited. This is consistent with the dynamics on a PES having a "late barrier" to dissociation. The distance between thresholds are approximately $50 \mathrm{~kJ} / \mathrm{mol}$ which is higher than the energy in a vibrational quantum $(35 \mathrm{~kJ} / \mathrm{mol})$. This is not consistent with the dynamics on a single two-dimensional PES. However, the experimental data cannot in any way be satisfactorily fit without increasing the threshold separation beyond that given by the vibrational energy.

The enlarged threshold separation found when applying this simplified state resolved empirical model can be explained as a natural consequence of the true multidimensionality of the real system. ${ }^{13}$ In contrast to dynamics on a single two-dimensional PES, the beam experiments probe an aver- 


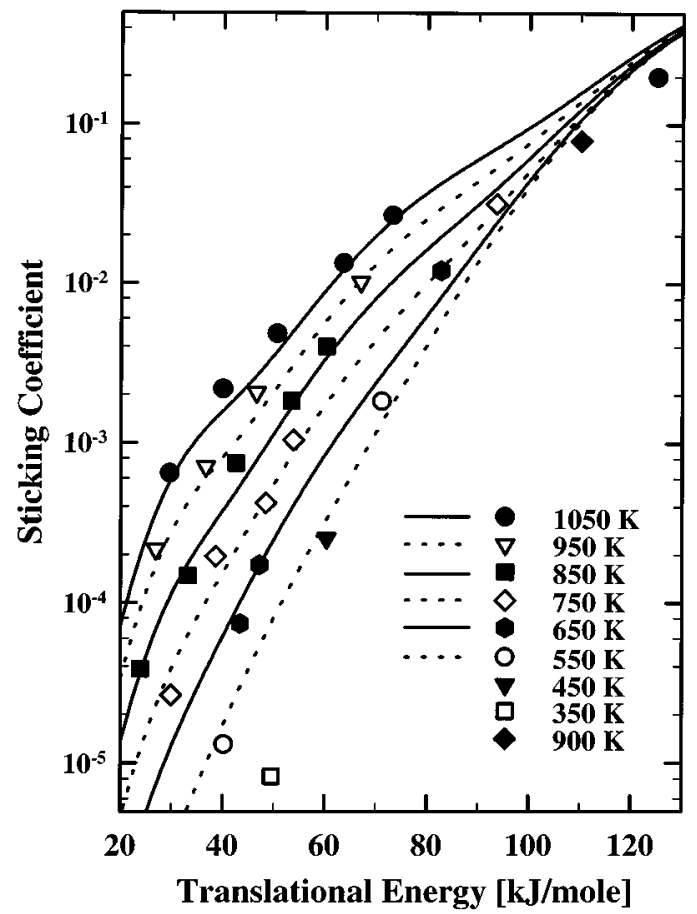

FIG. 9. Initial sticking coefficient as function of translational energy and vibrational temperature as indicated by the symbol legend. Symbols are measured sticking coefficients corrected for velocity distributions in the molecular beams as described in the text. The resulting fit is shown by alternating solid and dashed lines.

age sticking probability over the unit cell and impact orientation of the methane. These and other degrees of freedom can qualitatively be accounted for by introducing a distribution of 2D barriers. This distribution of barriers is of course the same for all the considered vibrational states and will tend to increase the thresholds for the sticking. However, the magnitude of the threshold shift will decrease with increasing vibrational excitation as explained in more detail in the following paper. ${ }^{13}$ In this paper the isotope effect observed in the present molecular beam study is also explained as mainly due to changes in zero point energy of $\mathrm{R}-\mathrm{D}$ relative to $\mathrm{R}-\mathrm{H}$.

The deviation from normal-energy scaling observed in this molecular beam study is a quite reasonable effect of a distribution of barriers. Recently, it has been shown by wave packet calculations that energetic and geometric corrugation in the PES lead to opposing deviations from normal-energy scaling. ${ }^{20}$ The reduced sticking at off-normal incidence observed in this study is then indicative of dominating energetic corrugation.

There are other theoretical approaches to the dissociative sticking of methane on metals. Ab initio calculations of the transition state on clusters combined with transition state theory is one approach. ${ }^{21}$ Another approach is a statistical mechanism for dissociation proposed by Ukraintsev et al. ${ }^{22}$ where the methane is strongly coupled to a local cluster of surface atoms forming an activated collision complex which decays according to Rice-Ramsperger-Kassel-Marcus (RRKM) rate theory. This model was used to fit the disso- ciative sticking of methane on $\operatorname{Pt}(111)$ and may also fit the experimental data of this work.

The huge effect of vibrational energy observed in the present molecular beam study supports the suggestion in Ref. 11 that this could be a possible reason for the discrepancy between the previous molecular beam study of $\mathrm{CH}_{4}$ on $\mathrm{Ni}(100)^{3}$ and the direct measurement of the thermal sticking coefficient when the direct mechanism is assumed. However, in the present detailed molecular beam study, we measure sticking coefficients much lower than in the previous study. ${ }^{3}$ In the present work the dynamical importance of translational energy and vibrational excitation are investigated separately. An empirical state resolved model is established based on S-shaped curves describing the sticking probability as a function of translational energy for molecules in vibrational levels $\nu=0,1$, and 2. Assuming normal-energy scaling which was approximately observed in this work, it is straightforward with this model to calculate the thermal sticking coefficient by convoluting the model with the respective distributions of a gas in thermal equilibrium with the crystal surface. Figure 11 shows an Arrhenius plot of these calculated thermal sticking coefficients using normalenergy scaling. On the same figure is also shown the direct measurement of Chorkendorff et al. ${ }^{8}$ for the $\mathrm{Ni}(100)$ surface. The agreement between the measurements and the thermal sticking coefficient calculated from the molecular beam results is seen to be excellent. Any better agreement cannot be expected since the extrapolation of the state resolved sticking to low translational energy using the ansatz (5) can only be qualitative. Furthermore, the dependence on surface temperature is not included. The calculated thermal sticking coefficient can be partitioned into contributions from the vibrational states. It is seen that in the temperature range of interest in connection with catalytic steam reforming of methane $(\sim 1000 \mathrm{~K})$ the thermal sticking coefficient is dominated by contributions from vibrationally excited molecules. Also it is seen that the thermal sticking coefficient obtained from this molecular beam study does only depend linearly on the inverse temperature over limited temperature intervals. This suggests that there can be problems when thermal sticking coefficients obtained from bulb experiments at low temperatures are extrapolated linearly in an Arrhenius plot to the

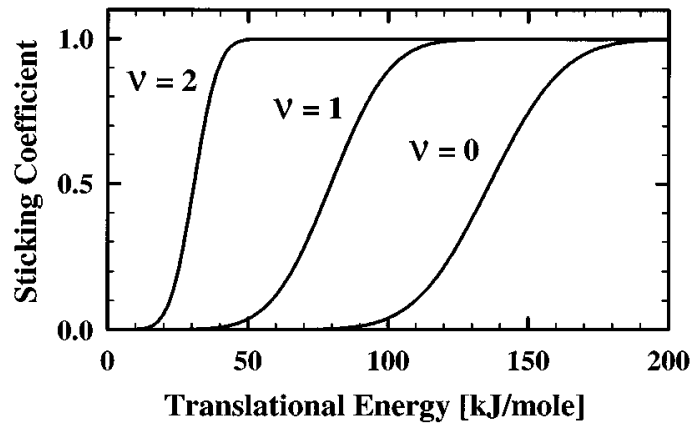

FIG. 10. The resulting sticking curves obtained using the model described in the text. Solid lines show the sticking coefficient as function of translational energy for $\mathrm{CH}_{4}$ in quasidiatomic vibrational excitations $\nu=0,1$, and 2 . 


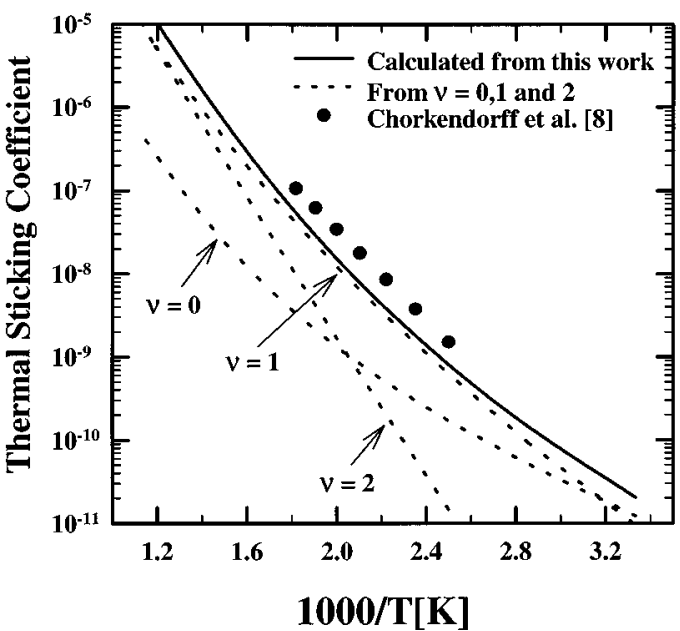

FIG. 11. Arrhenius plot of the calculated thermal sticking coefficient (solid line) obtained from this molecular beam study using the model described in the text. Solid circles are the measurements of Chorkendorff et al. (Ref. 8). Dashed lines show the contributions from $\mathrm{CH}_{4}$ in the vibrational excitations $\nu=0,1$, and 2 of the methane stretch modes as indicated by the arrows.

temperature range of interest in industrial catalysis.

Recently, it has been claimed that the $\mathrm{CH}_{4}$ sticking dynamics on $\mathrm{Ni}(100)$ at industrial catalytic conditions is dominated by a precursor mechanism. ${ }^{23}$ However, the molecular beam results presented here strongly suggests that the process is direct dissociative chemisorption since the agreement with the direct measurements of the thermal sticking coefficient is very good. Furthermore, in contrast to the work of Campbell et al. ${ }^{23}$ bulb experiments recently carried out at our laboratory using the same UHV apparatus and $\mathrm{Ni}(100)$ sample as used in the present molecular beam study, supports the mechanism of direct dissociative chemisorption. These experiments will be reported separately. ${ }^{9}$

\section{CONCLUSION}

This paper reports a detailed molecular beam study of the dissociative sticking of methane on $\mathrm{Ni}(100)$. The dissociation dynamics show all the characteristics of activated direct chemisorption. A dramatic activation by translational energy as well as vibrational excitation is observed. The initial sticking coefficient increases 2-3 orders of magnitude as the translational energy for a fixed vibrational energy is raised from 20 to $120 \mathrm{~kJ} / \mathrm{mol}$. At a fixed translational energy of 40 $\mathrm{kJ} / \mathrm{mol}$ the sticking coefficient increases two orders of magnitude as the vibrational temperature is raised from 550 to $1050 \mathrm{~K}$. Normal energy scaling is only approximately observed, the off-normal sticking systematically being slightly lower. The effect of surface temperature is observed to be weak at the experimental conditions. An isotope effect of approximately a factor $5-10$ is observed, $\mathrm{CH}_{4}$ being more reactive than $\mathrm{CD}_{4}$ at the same translational energy.
All observations qualitatively exhibits the features of the model proposed by Luntz et al., ${ }^{12,13}$ the strong dependence on vibrational temperature being a consequence of a barrier to dissociation mainly located in the vibrational coordinates of the PES governing the sticking dynamics. An extensive comparison of this model to the present molecular beam study is given in the following paper of this issue. ${ }^{13}$

Simplified modeling in terms state resolved sticking curves, considering only the vibrational $\mathrm{C}-\mathrm{H}$ stretch modes of methane as relevant for the sticking, provides a good description of the data. These sticking curves enables a calculation of the thermal sticking coefficient. The magnitude and activation energy of the calculated thermal sticking coefficient is found to be in excellent agreement with the bulb experiment of Chorkendorff et al. ${ }^{8}$ directly probing this. It is therefore suggested that also under isothermal conditions the sticking is direct dissociative chemisorption.

\section{ACKNOWLEDGMENTS}

We would like thank Professor Allan C. Luntz for many helpful comments and discussions. Also Bjørn Ølgaard Nielsen is acknowledged for his assistance in the laboratory. The present work was supported by the Danish Center for Surface Reactivity.

${ }^{1}$ J. R. Rostrup-Nielsen, Catalytic Steam Reforming (Springer, Berlin, 1984).

${ }^{2}$ M. B. Lee, Q. Y. Yang, and S. T. Ceyer, J. Chem. Phys. 87, 2724 (1987).

${ }^{3}$ A. V. Hamza and R. J. Madix, Surf. Sci. 179, 25 (1987).

${ }^{4}$ A. C. Luntz and D. S. Bethune, J. Chem. Phys. 90, 1274 (1989).

${ }^{5}$ C. T. Rettner, H. E. Pfnur, and D. J. Auerbach, Phys. Rev. Lett. 54, 2716 (1985).

${ }^{6}$ C. T. Rettner, H. E. Pfnur, and D. J. Auerbach, J. Chem. Phys. 84, 4163 (1986)

${ }^{7}$ T. P. Beebe, Jr., D. W. Goodman, B. D. Kay, and J. T. Yates, J. Chem. Phys. 87, 2305 (1987).

${ }^{8}$ I. Chorkendorff, I. Alstrup, and S. Ullmann, Surf. Sci. 227, 291 (1990).

${ }^{9}$ B. Ølgaard Nielsen, A. C. Luntz, P. M. Holmblad, and I. Chorkendorff, Catal. Lett. (accepted).

${ }^{10}$ L. Hanley, Z. Xu, and J. T. Yates, Jr., Surf. Sci. Lett. 248, L265 (1991).

${ }^{11}$ A. G. Sault, and D. W. Goodmann, J. Chem. Phys. 88, 7232 (1988).

${ }^{12}$ A. C. Luntz and J. Harris, Surf. Sci. 258, 397 (1991).

${ }^{13}$ A. C. Luntz, J. Chem. Phys. 102, 8264 (1995).

${ }^{14}$ N. T. Andersen, F. Topsøe, I. Alstrup, and J. R. Rostrup-Nielsen, J. Catal. 104, 454 (1987); I. Alstrup and N. T. Andersen, ibid. 104, 466 (1987).

${ }^{15}$ C. Klink, L. Olesen, F. Besenbacher, I. Stensgaard, E. Lægsgaard, and N. Lang, Phys. Rev. Lett. 71, 4350 (1993).

${ }^{16}$ J. H. Onuferko, D. P. Woodruff, and B. W. Holland, Surf. Sci. 87, 357 (1979).

${ }^{17}$ D. A. King and M. G. Wells, Surf. Sci. 29, 454 (1972).

${ }^{18} \mathrm{G}$. Scoles, Atomic and Molecular Beam Methods (Oxford University, London, 1988).

${ }^{19}$ H. A. Michelsen, C. T. Rettner, D. J. Auerbach, and R. N. Zare, J. Chem. Phys. 98, 8294 (1993).

${ }^{20}$ G. R. Darling and S. Holloway, Surf. Sci. Lett. 304, L461 (1994).

${ }^{21}$ H. Burghraef, A. P. J. Jansen, and R. A. van Santen, J. Chem. Phys. 98, 8810 (1993)

${ }^{22}$ V. A. Ukraintsev and I. Harrison, J. Chem. Phys. 101, 1564 (1994).

${ }^{23}$ R. A. Campbell, J. Szanyi, P. Lenz, and D. W. Goodman, Catal. Lett. 17, 39 (1993). 